\title{
Revised Structures of Dehydrostenines A and B: Total Syntheses of $( \pm)$-Dehydrostenine $A$ and Structure Assigned to Dehydrostenine B
}

\author{
Wesley J. Olivier, Nigel T. Lucas, Alex C. Bissember, ${ }^{*}$ Jason A. Smith, ${ }^{*}$
}

\begin{abstract}
The first total syntheses of the Stemona alkaloid dehydrostenine $A$ and the structure assigned to dehydrostenine $B$ have been completed from a simple pyrrole substrate in 10 and 11 steps, respectively. Two independent Brønsted-acid-mediated intramolecular Michael additions were exploited to construct the tetracyclic dehydrostenine core. As a result of our synthetic studies and associated analysis of the relevant literature, we propose revisions of the structures originally assigned to dehydrostenines $A$ and $\mathrm{B}$.
\end{abstract}

The Stemona alkaloids are a class of diverse polycyclic natural products isolated from plants belonging to the genus Stemona. The structures of many Stemona alkaloids feature a bicyclic pyrrolo[1,2-a]azepine core in addition to several other fused or otherwise attached rings. Examples, such as stenine (1) and sessilifoliamide C (2), were first isolated from Stemona tuberosa and Stemona sessilifolia, respectively (Figure 1) $)^{1,2}$ Stenine (1) has received particular attention as a target for total synthesis, with over 25 reported total or formal syntheses of this natural product completed to date..$^{3,4}$ Stenine-type alkaloids are known to exhibit a variety of biological activities and stenine possesses significant acetylcholinesterase activity, for example. ${ }^{5}$

Dehydrostenines A (3) and B (4) are stenine-type alkaloids in the pyrrole oxidation state, which were isolated from a plant species classified as Stemona sessilifolia in 2017 and it appeared that these molecules had not yet succumbed to synthesis when we started our study. ${ }^{6}$ The publication detailing the isolation and characterization of these natural products assigned the structure of dehydrostenine $A$ as compound 3 (Figure 1). The stereochemical assignment of the C9-carbon was derived from conclusions drawn from NOESY correlations. Interestingly, the same report included the $\mathrm{X}$-ray crystal structure of this natural product, which instead revealed a structure consistent with the C9- epimer (i.e., structure 5). ${ }^{6}$ Given these issues, we wanted to clarify this discrepancy by validating the structure of dehydrostenine A (5) via total synthesis. We were also interested

[*] Mr W. J. Olivier, Assoc. Prof. A. C. Bissember, Assoc. Prof. J. A. Smith

School of Natural Sciences - Chemistry, University of Tasmania Hobart, Tasmania, Australia

E-mail: Alex.Bissember@utas.edu.au, Jason.Smith@utas.edu.au

Assoc. Prof. N. T. Lucas

Department of Chemistry, University of Otago, Dunedin, Otago, New Zealand

Supporting information and the ORCID identification number(s) for the author(s) of this article can be found under: https ://doi.org/10 in preparing the reported structure of dehydrostenine B (4), as this would allow us to corroborate the structural and stereochemical assignments for this natural product that were inferred from 1D and 2D NMR spectroscopic data. ${ }^{6}$
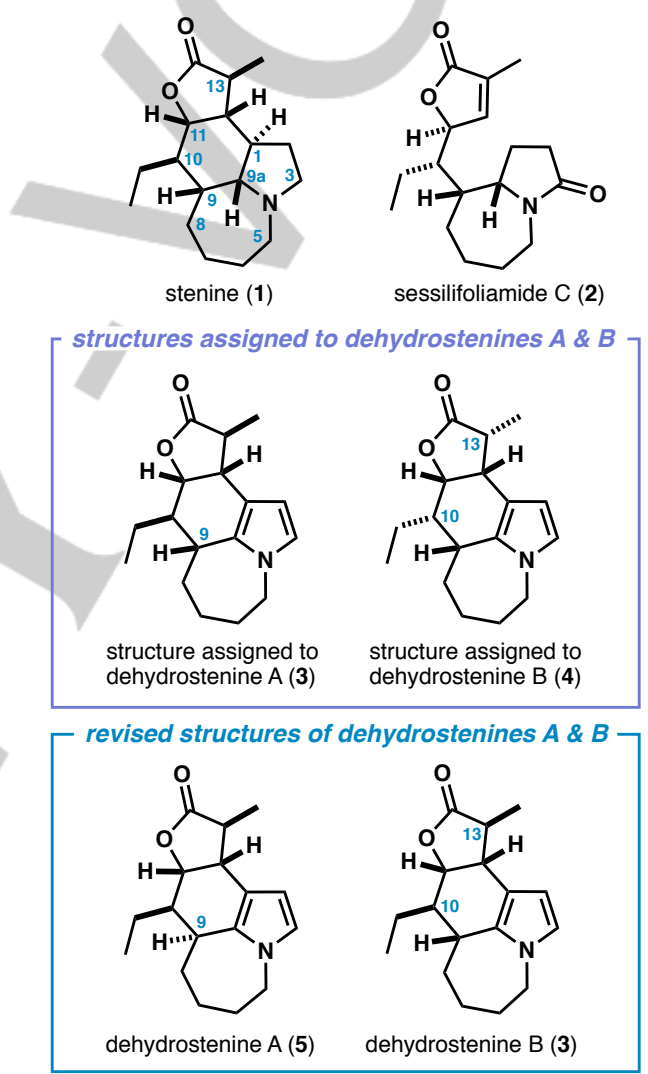

Figure 1. Examples of Stemona alkaloids.

We recently reported unified total syntheses of sessilifoliamides C (2), B, and D from a pyrrole substrate. ${ }^{7}$ Because these bicyclic natural products feature structural similarities to dehydrostenine $A$, we anticipated that we could access structure $\mathbf{5}$ by germane chemistry. Specifically, we proposed that pyrrole 7 , which bears a pendant $\alpha, \beta$-unsaturated lactone, could be accessed from a common (early-stage) pyrrole intermediate from which we prepared the sessilifoliamides (Figure 2). ${ }^{7}$ Furthermore, we posited that lactone 7 could be treated with a Brønsted acid to construct the tetracyclic dehydrostenine framework (6), via an intramolecular conjugate addition. ${ }^{8}$ This would represent an original general approach to accessing the tetracyclic core of stenine-type alkaloids. BookerMilburn and coworkers employed a somewhat similar end-game strategy to forge the dehydrostenine core that involved selective

For internal use, please do not delete. Submitted Manuscript 
protonation and cyclization of a pyrrole tethered to 2silyloxyfuran. ${ }^{9}$ However, the authors' experimental observations suggest that this cyclization proceeds via a silylated lactone (and not a protonated lactone) intermediate. ${ }^{9}$ An unrelated approach for the construction of the dehydrostenine framework has also been reported. ${ }^{10}$ By design, our strategy focused on developing a pathway that would also allow for the divergent synthesis of the structure assigned to dehydrostenine B (4) for the reasons outlined earlier.

Herein, we report the first total synthesis of dehydrostenine A (5) and propose its revised structure. In addition, we synthesized the structure originally assigned to dehydrostenine B (4). ${ }^{6}$ Consistent with our findings and analysis of relevant and complementary synthetic studies by Booker-Milburn and coworkers, ${ }^{9}$ we propose the revised structure of dehydrostenine $\mathrm{B}(3)$.

Because our proposed approach to assembling tetracycle 6 was unprecedented, prior to embarking on our synthesis of dehydrostenine $A(\mathbf{5})$, we evaluated the viability of the centerpiece intramolecular Michael addition using model C-10 desethyl substrate 8 (see Supporting Information). ${ }^{11}$ This provided the corresponding tetracyclic pyrrole efficiently and exclusively in only 4 steps from 10, which galvanized us to apply this strategy to target natural product $\mathbf{5}$. Our synthesis commenced from commercially available pyrrole $\mathbf{9}$, which was converted to pyrroloazepine 10 in two steps and $59 \%$ yield on a two-gram scale as we have previously reported (Scheme 1). ${ }^{7} \alpha$-Ethylation of ester 10 provided pyrroloazepines $11 \mathrm{a}$ and $11 \mathrm{~b}$ in a $3.5: 1$ ratio in $77 \%$ combined yield employing optimized conditions. We also explored whether diastereoselective ethylation of ester 10 might be better facilitated from a derivative featuring a chiral auxiliary, however, this was not successful (see Supporting Information). The respective relative configurations of previously reported compounds $11 \mathrm{a}$ and $11 \mathrm{~b}^{7}$ were tentatively assigned on the basis that major diastereomer 11a would derive from delivery of iodoethane to the less hindered face of the enolate derived from substrate 10.
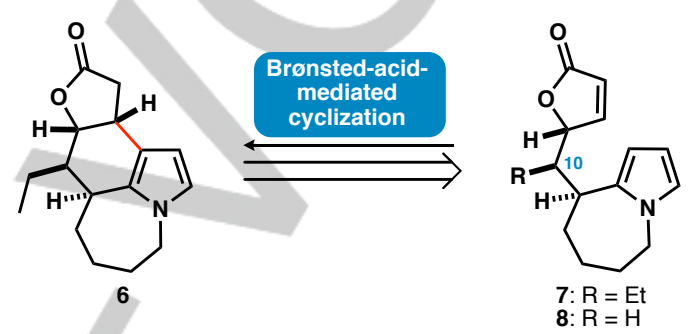

Figure 2. Strategy for constructing the tetracyclic core of dehydrostenine A (5).

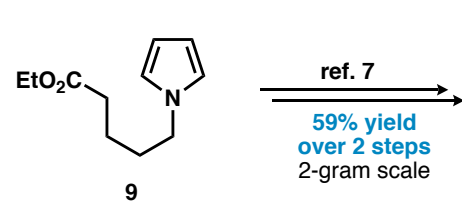

9

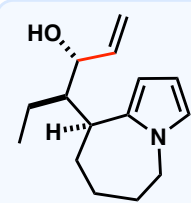

$( \pm)-14 a$ ( $48 \%$ yield over 2 steps)

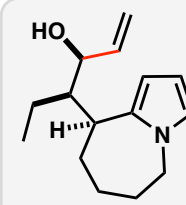

( \pm )-14b ( $8 \%$ yield over 2 steps)

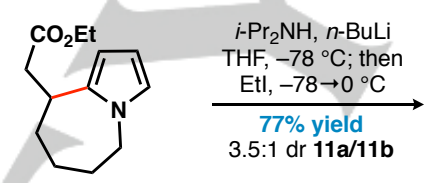

$( \pm)-10$

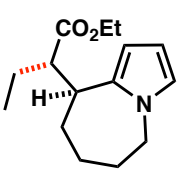

$( \pm)-11 b$

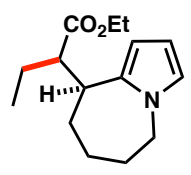

$( \pm)-11 \mathrm{a}$

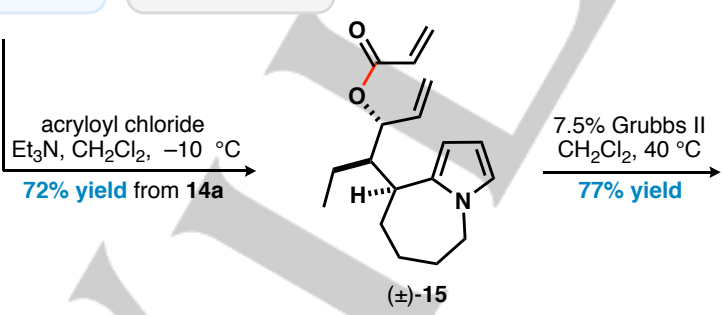

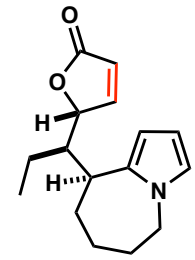

(土)-7

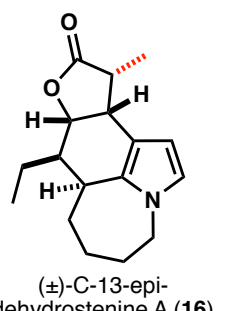

dehydrostenine A (16)

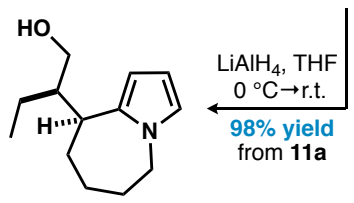

$( \pm)-12$
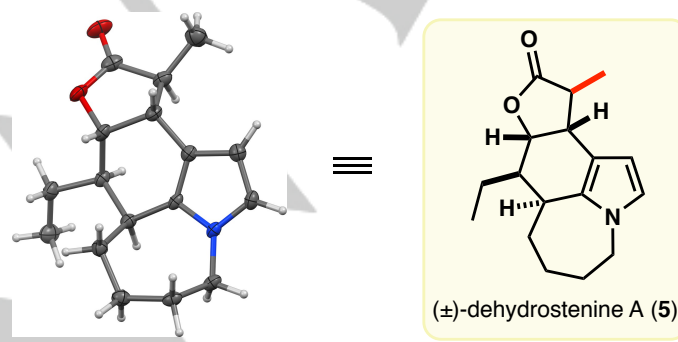

( \pm )-dehydrostenine A (5)

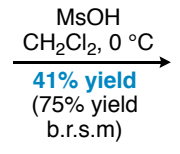

LiHMDS, THF, $-78{ }^{\circ} \mathrm{C}$

then Mel, $-78 \rightarrow-20^{\circ} \mathrm{C}$

$54 \%$ yield, 5.7:1 dr 5/16

Scheme 1. Total synthesis of dehydrostenine A (5) from commercially available pyrrole $\mathbf{9}$.

For internal use, please do not delete. Submitted Manuscript 
We intended to selectively reduce ester $11 \mathrm{a}$ to aldehyde 13 and anticipated that this could be achieved directly via treatment with DIBAL-H. However, alcohol 12 was the only product obtained under these conditions. ${ }^{12}$ These issues prompted us to reduce ester 11a to alcohol 12 with $\mathrm{LiAlH}_{4}$ and obtain aldehyde 13 by oxidation. Because hypervalent iodine reagents, such as 2iodoxybenzoic acid (IBX) and Dess-Martin periodinane, do not offer the required chemoselectivity in the presence of pyrroles, ${ }^{7,13}$ we attempted to prepare aldehyde 13 via standard Swern conditions. Although this resulted in decomposition, a ParikhDoering oxidation successfully delivered aldehyde $\mathbf{1 3 .} .^{14,15}$ Product 13 was immediately treated with vinylmagnesium bromide to furnish vinyl alcohols $14 a$ and $14 b$ in a $4: 1$ ratio and $56 \%$ combined yield over two steps. ${ }^{16}$ The respective relative configurations of $14 \mathrm{a}$ and $14 \mathrm{~b}$ were tentatively assigned with reference to the stereoselectivity observed in the reaction of vinylmagnesium bromide with the pyrrolidinone analogue of aldehyde $13 .{ }^{7}$ Major diastereomer $14 a$ is consistent with delivery of the nucleophile to the less hindered face of aldehyde 13.

O-Acylation of alcohol 14a with acryloyl chloride, followed by ring-closing metathesis (RCM) with Grubbs second generation catalyst furnished $\alpha, \beta$-unsaturated lactone 7 in $55 \%$ yield over the two steps. $\mathrm{MsOH}$-mediated annulation of lactone 7 furnished tetracycle 6 exclusively in $41 \%$ yield $(75 \%$ yield based on recovered starting material). ${ }^{17}$ We propose that the observed cisselectivity derives from the higher ring strain associated with the trans-fused analogue of lactone 6, which disfavors formation of the latter diastereoisomer. Comprehensive NMR analysis of tetracycle 6 supported our tentative assignments regarding the relative configurations of synthetic precursors. ${ }^{9}$ Subsequent methylation completed the synthesis of target 5 and C13-epimer 16 in a 5.7:1 ratio. We confirmed structure 5 by single crystal Xray crystallography. ${ }^{18}$ The spectroscopic and crystallographic data obtained for compound $\mathbf{5}$ were consistent with equivalent data for the isolated natural product (see Supporting information). ${ }^{6}$

It should be noted that the structure originally assigned to dehydrostenine A (3) was synthesized by Booker-Milburn and coworkers. These researchers also secured the relative configuration of this structure via single crystal $X$-ray crystallography of C3-formylated derivative 17 (Figure 3). ${ }^{9}$ As expected, the spectroscopic data for isolated dehydrostenine $A$ is not consistent with equivalent data for structure 3 reported by
Booker-Milburn and coworkers (see Supporting Information). ${ }^{6,9}$ When these findings are taken together, it is evident that the correct structure of dehydrostenine A is molecule 5 (i.e., the C9epimer of the originally assigned structure (3). ${ }^{6}$

Interestingly, through this process we also noted that the NMR spectroscopic data for aforementioned structure 3 reported by Booker-Milburn and co-workers conspicuously matched equivalent data reported for dehydrostenine $B$ in the original isolation study (see Supporting Information). ${ }^{6,9}$ This revealed that the structure originally assigned to dehydrostenine B (4) was also incorrect. Furthermore, it highlighted that Booker-Milburn and coworkers accomplished the first total synthesis of dehydrostenine B (3) in $2019 .^{9}$

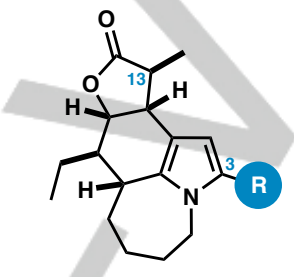

$( \pm)-3: \quad \mathrm{R}=\mathrm{H}$ $( \pm)-17: \mathrm{R}=\mathrm{CHO}$

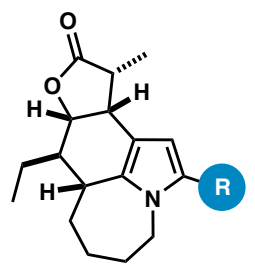

$( \pm)-18 a: R=H$ $( \pm)-18 b: R=C H O$
Figure 3. Compounds 3, 17, and 18 synthesized by Booker-Milburn and coworkers; structures 17 and $18 \mathrm{~b}$ were secured by single crystal X-ray crystallography. ${ }^{9}$

We aimed to prepare target $\mathbf{4}$ to gain further evidence that the structure assigned to dehydrostenine B (4) was incorrect. Our initial attempt commenced from major vinyl alcohol diastereoisomer 14a (Scheme 2). This involved the $\mathrm{S}_{\mathrm{N}} 2$ substitution of major vinyl alcohol 14a with methacrylic acid under Mitsunobu conditions, which inverted the C11-stereocenter, followed by RCM. The Mitsunobu reaction was particularly inefficient. ${ }^{19}$ Unfortunately, the key intramolecular Michael addition did not furnish target 4 . In the presence of either $\mathrm{MsOH}$ and $\mathrm{TfOH}$, we did not detect the cyclization product and only decomposition of substrate $\mathbf{2 0}$ was observed. ${ }^{20,21}$ This suggested that a more viable route to the structure assigned to dehydrostenine B (4) would involve cyclization from a substrate analogous to heterocycle 7 that did not feature the methyl group.

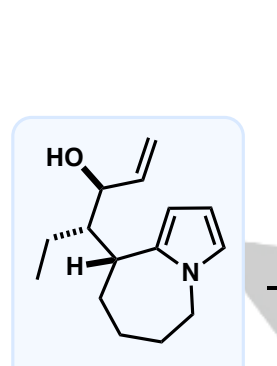

$( \pm)-14 a$

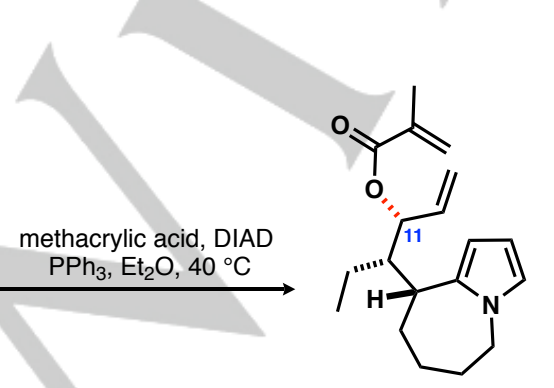

$( \pm)-19$

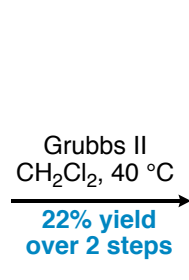

over 2 steps

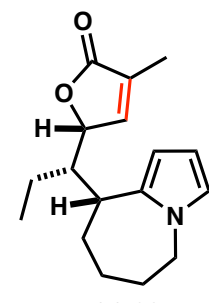

$( \pm)-20$
$\mathrm{MsOH}$ or $\mathrm{TfOH}$ $\mathrm{CH}_{2} \mathrm{Cl}_{2}, \leq 25^{\circ} \mathrm{C}$

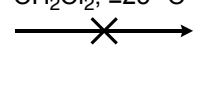

$( \pm)-4$

Scheme 2. Attempted synthesis of the structure assigned to dehydrostenine B (4) from major diastereomer 14a. 
Our revised attempt to access the structure assigned to dehydrostenine B (4) employed minor vinyl alcohol diastereoisomer $14 \mathrm{~b}$ and followed an analogous route to the one that delivered dehydrostenine A (5) from 14a (Scheme 3). Thus, O-acylation of alcohol 14b with acryloyl chloride and RCM of ensuing acrylate ester $\mathbf{2 1}$ provided furanone $\mathbf{2 2}$ in $\mathbf{5 6 \%}$ yield over 2 steps. $\alpha, \beta$-Unsaturated lactone 22 was treated with $\mathrm{MsOH}$ to afford tetracycle $\mathbf{2 3}$ in high yield. Methylation of substrate $\mathbf{2 3}$ exclusively furnished heterocycle $\mathbf{2 4}$, which is the C13-epimer of target 4. In comparison, methylation reactions performed on diastereomers of molecule $\mathbf{2 3}$ (6 6 and the desmethyl derivative of 3) give mixtures of C13-epimers. ${ }^{9}$ We anticipate that the high selectivity of this alkylation stems from the exclusive delivery of the methyl group from the less hindered $\beta$-face of the enolate derived from molecule 23 as the $\alpha$-face of this structure is essentially inaccessible (Figure 4).

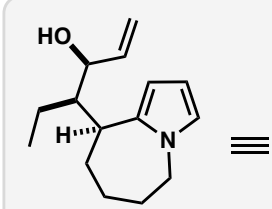

$( \pm)-14 b$

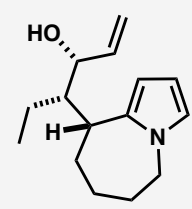

$( \pm)-14 b$

acryloyl chloride, $\mathrm{Et}_{3} \mathrm{~N}$ $\underset{56 \% \text { yield }}{\stackrel{\mathrm{CH}_{2} \mathrm{Cl}_{2},-15{ }^{\circ} \mathrm{C}}{\longrightarrow}}$

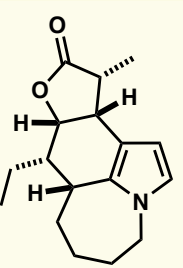

$( \pm)-4$ : structure assigned to dehydrostenine $B$

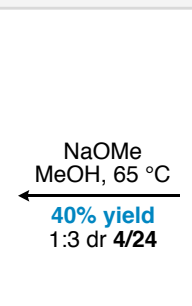

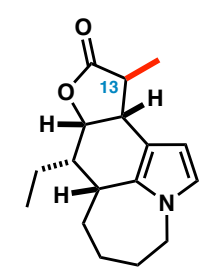

$( \pm)-24$

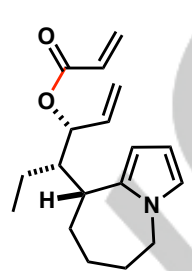

$( \pm)-21$

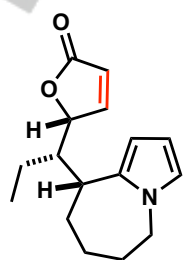

$( \pm)-22$

Scheme 3. Synthesis of the structure assigned to dehydrostenine B (4) from minor diastereomer 14b

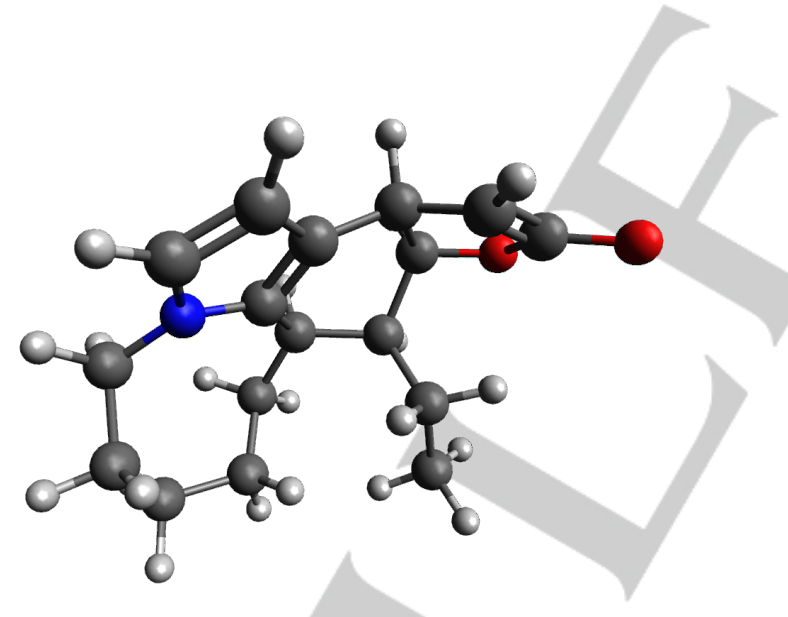

Figure 4. Simulated molecular representation of the enolate derived from substrate 23.

We attempted to epimerize compound 24 under basic conditions at (or below) ambient temperature, employing LiHMDS, $\mathrm{NaOMe}$, and $\mathrm{KH}$, respectively. ${ }^{22}$ Unfortunately, each of these experiments only returned C13-epimer 24 . We propose that the C10-ethyl group effectively shields the $\alpha$-face of the bowl-shaped substrate to block deprotonation and, hence, the required enolate cannot be generated at low temperature. However, heating molecule 24 with $\mathrm{NaOMe}$ in methanol delivered the structure assigned to dehydrostenine $B(4)$ and substrate 24 in a 1:3 ratio.
As we anticipated, the spectroscopic data we obtained for compound $\mathbf{4}$ were not consistent with equivalent data for isolated dehydrostenine B (see Supporting information). ${ }^{6,23}$ As we noted earlier, NMR spectroscopic data for structure 3 reported by Booker-Milburn and co-workers clearly matches equivalent data reported for isolated dehydrostenine $\mathrm{B},{ }^{6,9}$ which demonstrates that these researchers accomplished the first total synthesis of dehydrostenine $B$ by a particularly elegant and efficient approach. ${ }^{9}$ When our work and the aforementioned complementary synthetic studies undertaken by Booker-Milburn and coworkers are taken together, we propose that the correct structure of dehydrostenine $B$ is molecule 3 .

In conclusion, we have completed the first total syntheses of the Stemona alkaloid dehydrostenine $\mathrm{A}$ and the structure assigned to dehydrostenine $B$ in 10 and 11 steps, respectively. The tetracyclic core of these alkaloids was prepared via an endgame strategy involving a conjugate addition of a pyrrole onto a pendant $\alpha, \beta$-unsaturated lactone. As a result of our investigation, we have addressed the stereochemical discrepancy in the literature regarding dehydrostenine $A$ and have reassigned the relative configuration of dehydrostenine $B$ in accordance with our findings and analysis of the relevant literature..$^{6,9,24}$

\section{Acknowledgements}

The authors thank the University of Tasmania School of Natural Sciences - Chemistry for funding, the University of Tasmania 
Central Science Laboratory for providing access to NMR spectroscopy services, and $\mathrm{Dr}$ Curtis Ho for assistance preparing Figure 4. W.J.O. thanks the Australian Government for a Research Training Program Scholarship. A.C.B.'s contributions were supported by an ARC Future Fellowship (FT200100049). N.T.L. acknowledges financial support by the MacDiarmid Institute for Advanced Materials and Nanotechnology, and the Marsden Fund Council, managed by the Royal Society Te Apārangi, New Zealand.

\section{Conflict of Interest}

The authors declare no conflict of interest.

Keywords: dehydrostenines $\bullet$ total synthesis $\cdot$ pyrrole $\cdot$ Stemona alkaloids

\section{References}

[1] S. Uyeo, H. Irie, H. Harada, Chem. Pharm. Bull. 1967, 15, 768-770.

[2] Y. Hitotsuyanagi, M. Hikita, T. Oda, D. Kakuta, H. Fukaya, K. Takeya, Tetrahedron 2007, 63, 1008-1013.

[3] For total syntheses of stenine published prior to 2010, see: (a) R. A. Pilli, M. C. F. de Oliveira, Nat. Prod. Rep. 2000, 17, 117-127. (b) R. A. Pilli, G. B. Rosso, M. C. F. de Oliveira, Nat. Prod. Rep. 2010, 27, 1908-1937.

[4] For selected total syntheses of stenine published since 2010, see: (a) J. Chen, J. Chen, Y. Xie, H. Zhang, Angew. Chem. Int. Ed. 2012, 51, 10241027. (b) H. Fujioka, K. Nakahara, N. Kotoku, Y. Ohba, Y. Nagatomi, T.S. Wang, Y. Sawama, K. Murai, K. Hirano, T. Oki, S. Wakamatsu, Y. Kita, Chem. - Eur. J. 2012, 18, 13861-13870. (c) J. Chen, Y. Xie, Zhang, H Tetrahedron 2015, 71, 3747-3755.

[5] H. Greger, Phytochem. Rev. 2019, 18, 463-469.

[6] J. L. Dong, Z.-D. Yang, S.-Y. Zhou, H.-T. Yu, X.-J. Yao, H.-Y. Xue, Z.-M Shu, Phytochem. Lett. 2017, 19, 259-262.

[7] W. J. Olivier, A. C. Bissember, J. A. Smith, Org. Lett. 2021, 23, 34373441.

[8] W. J. Olivier, M. G. Gardiner, A. C. Bissember, J. A. Smith, Tetrahedron 2018, 74, 5436-5441.

[9] R. L. Connelly, J. P. Knowles, K. I. Booker-Milburn, Org. Lett. 2019, 21, 18-21.

[10] R. W. Bates, S. Sridhar, J. Org. Chem. 2011, 76, 5026-5035.

[11] Substrate 8 was one of a number of pendant Michael-acceptors that we evaluated in this transformation. However, $\alpha, \beta$-unsaturated lactone 8 was the only system that allowed us to construct the core of the target framework efficiently.

[12] In contrast, treatment of ester 10 (the desethyl analogue of ester 11a) with DIBAL-H successfully provided the corresponding aldehyde (see Supporting Information).

[13] (a) J. K. Howard, C. J. T. Hyland, J. Just, J. A. Smith, Org. Lett. 2013, 15, 1714-1717. (b) I. A. Andreev, N. K. Ratmanova, A. M. Novoselov, D. S Belov, I. F. Seregina, A. V. Kurkin, Chem. - Eur. J. 2016, 22, 7262-7267.

[14] Similar observations concerning the utility of Swern and Parikh-Doering oxidations were made by Booker-Milburn and coworkers in related studies (ref. 9)
[15] The oxidation of alcohol 12 to rather unstable aldehyde 13 was more efficient and reproducible when performed on a 150-200 $\mathrm{mg}$ scale in comparison to experiments performed on larger scales (>500 mg).

[16] Products $14 \mathbf{a} / \mathbf{1 4} \mathrm{b}$ were formed in $3: 1 \mathrm{dr}$ when the Grignard addition was conducted at $0{ }^{\circ} \mathrm{C}$. We thought we could convert $14 \mathrm{a}$ to $14 \mathrm{~b}$ via oxidation to the ketone and subsequent reduction, however, this route was not feasible (see Supporting Information).

[17] We observed that molecule 6 is prone to decomposition under the reaction conditions over prolonged periods of time. It is likely that the slow reaction of $\mathbf{7}$ to $\mathbf{6}$, compared to the efficient reaction of $\mathbf{2 2}$ to $\mathbf{2 3}$ (vide infra) derives from the significant differences in the respective threedimensional structures of more planar tetracycle $\mathbf{6}$ in comparison to bowlshaped tetracycle 23.

[18] CCDC 2091223 contains the supplementary crystallographic data for this paper. These data can be obtained free of charge from The Cambridge Crystallographic Data Centre.

[19] We investigated varying the temperature, solvent, stoichiometry of reagents/reactants, and concentration, but were unable to develop a more efficient Mitsunobu reaction with methacrylic acid and substrate 14a. We also conducted a Mitsunobu reaction with $\mathbf{1 4 a}$ and $p$ nitrobenzoic acid, which has been reported to increase the yield of this transformation with sterically hindered alcohols. While this modification greatly enhanced consumption of $\mathbf{1 4 a}$, the formation of by-products significantly compromised the efficiency of this route. For selected reports on the use of $p$-nitrobenzoic acid in Mitsunobu reactions see: (a) S. F. Martin, J. A. Dodge, Tetrahedron 1991, 32, 3017-3020. (b) J. A. Dodge, J. I. Trujillo, M. Presnell, J. Org. Chem. 1994, 59, 234-236.

[20] We observed similar patterns of reactivity in analogous intramolecular Michael additions of $\mathrm{N}$-tethered pyrrole substrates in synthetic studies targeting sessilifoliamide natural products. Thus, this somewhat unexpected attenuation of reactivity provided by $\alpha$-alkyl substituents within these systems likely has a common fundamental basis. Investigating this reactivity in more detail is the subject of future work.

[21] We expected protonation of the enol intermediate from the more accessible $\beta$-face would deliver compound 4 by this approach based on relevant observations from Booker-Milburn and coworkers (ref. 9).

[22] In the conversion of sessilifoliamide $B$ to sessilifoliamide $\mathrm{D}, \mathrm{NaOMe}$ (or $\mathrm{K}_{2} \mathrm{CO}_{3}$ in $\mathrm{MeOH}$ ) epimerized analogous $\alpha$-methyl lactones (ref. 7).

[23] For completeness, we compared the respective NMR spectroscopic data for other synthetic diastereomers of structure 4 (compounds 5 and 24) that we prepared in our study to equivalent data for isolated dehydrostenine B (ref. 6). We also compared the NMR spectroscopic data for diastereomer 18a synthesized by Booker-Milburn and coworkers to equivalent data for isolated dehydrostenine B (refs. 6 and 9). In each case, the NMR spectroscopic data did not match the reported data for dehydrostenine $B$ as expected (see Supporting Information).

[24] We contacted the corresponding authors of the study reporting the isolation of dehydrostenines $A$ and $B$ (ref. 6) who agreed with the revised structure of dehydrostenine $A(5)$ that we have proposed (personal communication with Prof. Z.-D. Yang, August 2021) 


\section{COMMUNICATION}

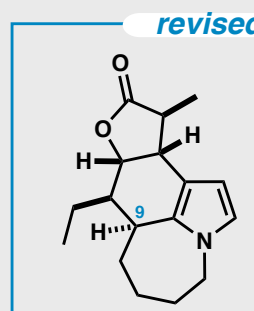

dehydrostenine A

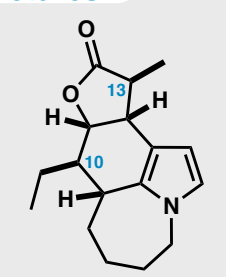

dehydrostenine B
Wesley J. Olivier, Nigel T. Lucas, Alex

C. Bissember* Jason A. Smith*

Page No. - Page No.

Revised Structures of Dehydrostenines

$A$ and B: Total Syntheses of ( \pm )-

Dehydrostenine $A$ and Structure

Assigned to Dehydrostenine B

The first total syntheses of the Stemona alkaloid dehydrostenine $A$ and the structure assigned to dehydrostenine $B$ have been completed from a simple pyrrole substrate in 10 and 11 steps, respectively. Two independent Brønsted-acid-mediated intramolecular Michael additions were exploited to construct the tetracyclic dehydrostenine core. As a result of our synthetic studies and associated analysis of the relevant literature, we propose revisions of the structures originally assigned to dehydrostenines $A$ and $B$. 